\section{(2) OPEN ACCESS}

\title{
Data-driven modelling of mutational hotspots and in silico predictors in hypertrophic cardiomyopathy
}

\author{
Adam Waring (10 , ${ }^{1}$ Andrew Harper (1) , ${ }^{1}$ Silvia Salatino, ${ }^{1}$ Christopher Kramer, ${ }^{2}$ \\ Stefan Neubauer, ${ }^{3}$ Kate Thomson, ${ }^{4}$ Hugh Watkins, ${ }^{1,3}$ Martin Farrall ${ }^{1,3}$
}

- Additional material is published online only. To view please visit the journal online (http://dx.doi.org/10.1136/ jmedgenet-2020-106922).

${ }^{1}$ Wellcome Centre for Human Genetics, University of Oxford, Oxford, UK

${ }^{2}$ Department of Medicine, University of Virginia, Charlottesville, Virginia, USA ${ }^{3}$ Radcliffe Department of Medicine, University of Oxford, Oxford, UK

${ }^{4}$ Oxford Medical Genetics Laboratories, Churchill Hospital, Oxford, UK

\section{Correspondence to}

Professor Martin Farrall, Wellcome Centre for Human Genetics, University of Oxford, Oxford, UK:

martin.farrall@cardiov.ox.ac.uk

Received 18 February 2020

Revised 17 June 2020

Accepted 20 June 2020

Published Online First 30 July 2020

Check for updates

(C) Author(s) (or their employer(s)) 2021. Re-use permitted under CC BY. Published by BMJ.

To cite: Waring A, Harper A, Salatino S, et al. J Med Genet 2021:58:556-564.

\section{ABSTRACT}

Background Although rare missense variants in Mendelian disease genes often cluster in specific regions of proteins, it is unclear how to consider this when evaluating the pathogenicity of a gene or variant. Here we introduce methods for gene association and variant interpretation that use this powerful signal.

Methods We present statistical methods to detect missense variant clustering ( $B / N$-test) combined with burden information (ClusterBurden). We introduce a flexible generalised additive modelling (GAM) framework to identify mutational hotspots using burden and clustering information (hotspot model) and supplemented by in silico predictors (hotspot+ model). The methods were applied to synthetic data and a case-control dataset, comprising 5338 hypertrophic cardiomyopathy patients and 125748 population reference samples over 34 putative cardiomyopathy genes.

Results In simulations, the BIN-test was almost twice as powerful as the Anderson-Darling or KolmogorovSmirnov tests; ClusterBurden was computationally faster and more powerful than alternative positioninformed methods. For 6/8 sarcomeric genes with strong clustering, Clusterburden showed enhanced power over burden-alone, equivalent to increasing the sample size by $50 \%$. Hotspot+ models that combine burden, clustering and in silico predictors outperform generic pathogenicity predictors and effectively integrate ACMG criteria PM1 and PP3 to yield strong or moderate evidence of pathogenicity for $31.8 \%$ of examined variants of uncertain significance.

Conclusion GAMs represent a unified statistical modelling framework to combine burden, clustering and functional information. Hotspot models can refine maps of regional burden and hotspot+ models can be powerful predictors of variant pathogenicity. The BIN-test is a fast powerful approach to detect missense variant clustering that when combined with burden information (ClusterBurden) may enhance disease-gene discovery.

\section{INTRODUCTION}

The clustering of pathogenic missense variants in specific regions or domains of proteins has been frequently reported. ${ }^{1-5}$ A plausible mechanism underpinning this phenomenon is the presence of multiple loss or gain-of-function variants within functionally important domains. ${ }^{6}$ Despite numerous examples of variant clustering, there have been few attempts to explicitly model variant residue position as a predictor of pathogenicity. ${ }^{7}$
Mendelian disease genes were historically identified by linkage and candidate gene studies in multiplex affected families. ${ }^{8}$ With technical advances in high-throughput, exome sequencing has become another approach to identify novel pathogenic genes and variants. The aggregated burden of rare variants in affected cases compared with healthy controls has proved to be a useful test to confirm candidate ${ }^{9}$ and identify novel, ${ }^{10}$ putative pathogenic genes. Several enhancements to this simple approach have been developed including weighting by variant frequency or functional annotation, ${ }^{11}$ integrating additional genetic risk factors such as polygenic risk scores ${ }^{12}$ or modelling both protective and deleterious variants by comparing variance in variant-level case-control frequencies. ${ }^{13} 14$ However, due to sample size limitations, few methods exist to test the rare disease ultrarare variant hypothesis in a case-control setting. Furthermore, there are no compelling examples where rare variants play a protective role. Here, we detect association based on a dominant model of rare deleterious variants and demonstrate that power can be increased by including variant residue position alongside gene-level burden. Unlike previous approaches to address this problem, ${ }^{715}$ we present computationally fast methods, for a realistic Mendelian disease genetic model, that place equal weight on the burden and clustering signals, making it a viable alternative strategy where simple burden testing has been unsuccessful.

The American College of Medical Genetics and Genomics (ACMG) has produced guidelines to interpret variant pathogenicity. ${ }^{16}$ These guidelines integrate diverse data and classify variants into five categories from benign to pathogenic. However, due to limited information available for many variants, they fall into the category 'variant of uncertain significance' (VUS). Although positional information is covered by criteria PM1 ('Located in a mutational hot spot and/or critical and wellestablished functional domain (eg, active site of an enzyme) without benign variation'), there is a lack of robust statistical evidence for mutational hotspots, resulting in inconsistent application of this criterion. Furthermore, although much work has gone into the development of in silico prediction scores, alternative scores can be conflicting, leading to discordance among testing laboratories ${ }^{17}$ and uncertainty in their application (criteria PP3: 'Multiple lines of computational evidence support a deleterious effect on the gene or gene product'). 
However, wherever large patient cohorts are attainable, mutational hotspots and the uncertainty surrounding in silico predictors can be directly estimated from the data.

Hypertrophic cardiomyopathy (HCM), a relatively common autosomal dominant disease (1 in 500 prevalence), is a major cause of heart disease in people of all ages ${ }^{18}$ and a cause of sudden cardiac death. In our cohort, eight sarcomeric genes collectively provide firm molecular diagnoses for $\sim 27 \%$ of HCM patients, with a further $\sim 13 \%$ of patients carrying a VUS in the same genes. It has been suggested that disease and genespecific approaches are needed to improve interpretation, ${ }^{19}$ and guidelines have been produced for specific genes and/or disease areas. ${ }^{20-23} \mathrm{HCM}$ is common enough to provide the large datasets needed for these gene-specific and data-driven approaches.

Here we propose new statistical approaches to explicitly include variant residue position in rare missense variant association and interpretation: BIN-test for detecting clusters of rare missense variants and ClusterBurden to combine this with burden information for association testing and generalised additive models $(G A M s)$ for hotspot estimation and modelling of in silico pathogenicity prediction algorithms. We apply these methods to a large cohort of $5338 \mathrm{HCM}$ patients and up to 125 $748 \mathrm{GnomAD}^{24}$ population controls. We demonstrate that using positional information increases power to detect disease-gene associations and elucidate the clustering signals present in 34 cardiomyopathy genes. We then use GAMs to model mutational hotspots and pathogenicity predictors for six core sarcomeric genes.

\section{METHODS}

\section{Patient cohorts and simulated data}

Next-generation sequence data for 34 cardiomyopathy genes (online supplementary table $\mathrm{S} 1$ ) were available from two large HCM cohorts (online supplementary method S1A); 2757 probands referred to the Oxford Medical Genetics Laboratory (OMGL) for genetic testing and 2636 probands recruited to the Hypertrophic Cardiomyopathy Registry (HCMR) project. ${ }^{25}$ Additional genome-wide SNP array data permitted exclusion of closely related individuals (ie, $\leq 3 \mathrm{rd}$ degree) for the HCMR cohort using the KING relationship inference software, ${ }^{26}$ and comparable data were unavailable to reliably identify closely related OMGL samples. High-coverage exonic sequences were captured by target enrichment and sequenced on the MiSeq platform (Illumina Inc). Joint bioinformatic processing of both datasets followed the Genome Analysis ToolKit version 4 best practice guidelines (online supplementary method S1B). OMGL variants were confirmed by Sanger sequencing, and HCMR variants were manually checked by inspection of BAM files.

The GnomAD exomes population reference database was used as a control group, which includes variant frequency data based on up to 125748 individuals. For both cases and controls, only missense variants with a GnomAD population maximum allele frequency of less than $0.0001^{910}$ were included. This excludes potentially common ancestry-specific variants that are unlikely to be pathogenic for HCM.

\section{Detecting missense variant burden and clustering: ClusterBurden}

Current methods to discover novel Mendelian disease genes focus on the burden of rare variants in an affected cohort relative to controls. Here we develop a powerful approach to detect differences in rare missense variant positions between two cohorts named BIN-test. We propose an approach that combines burden information (Fisher's exact test) with clustering signals (BIN-test) into a single framework: ClusterBurden. This framework tests the joint hypothesis of an excess of rare missense variants and differential clustering in case-control data. This was accomplished by combining the $\mathrm{p}$ values from a burden test with the BIN-test using Fisher's method. ${ }^{27}$ As there are no known examples of a protective burden of rare exonic variants in cardiomyopathy, we only consider an excess burden in the case group, making it a one-sided test. An important assumption of this method is that the contributing $\mathrm{p}$ values are independent; this was assessed in simulated data by Spearman's rank correlation test. ${ }^{28}$

As the background distribution of variant residue positions may be non-uniform, a cluster of variants observed in affected cases is insufficient to determine association with disease. Therefore, to detect disease-relevant clustering, distributions were compared between affected cases and unaffected controls. We propose BINtest to evaluate these distributional differences. First, the protein's linear sequence of amino acid residues is split into $k$ bins of equal length for each cohort. A $\chi^{2}$ two-sample test is applied to the resultant $k \times 2$ contingency table of binned variant counts. The null hypothesis is that the relative frequency of observed variants in each bin is the same for cases and controls. Significance depends on how many bins deviate from this expectation and by how much. We applied a $k \sim n^{2 / 5}$ heuristic ${ }^{29}$ to select the optimal number of bins $(k)$ dependent on $n$, the total number of observed variants. We compared the performance of the BIN-test with two other tests that compare distributions between two samples: AndersonDarling $(\mathrm{AD})^{30}$ and Kolmogorov-Smirnov (KS). ${ }^{31}$ Power and type 1 error were calculated using the $(r+1) /(n+1)$ estimator where $r$ represents the number of simulated datasets with $\mathrm{p}$ values less than 0.05 and $n$ is the number of simulations. ${ }^{32}$ To adjust for uneven sequencing coverage between the cohorts, the aggregated counts in each BIN-test bin were adjusted by the reciprocal of the mean $10 \times$ coverage across that bin for each cohort. For the burden test, sample sizes were similarly adjusted by the mean $10 \times$ coverage over the entire gene (online supplementary method S2).

To determine the theoretical performance of ClusterBurden, synthetic data were generated using a forward-time simulator (online supplementary method S3), designed to imitate rare variants in genes with discrete exonic regions of increased pathogenic potential. Six different scenarios were considered: three clustering scenarios (uniform, a single pathogenic cluster and multiple pathogenic clusters) and two protein lengths (500 and 1000 amino acid residues). For each scenario, 10000 synthetic datasets were generated with 5000 cases and 125000 controls. Variants were filtered by their frequency in simulated controls at a minimum allele frequency (MAF) of $<0.0001$. The performance of ClusterBurden was compared with two published positioninformed rare variant association tests (RVATs), DoEstRare ${ }^{7}$ and CLUSTER,${ }^{15}$ and three position-uninformed RVATs: C-alpha, ${ }^{14}$ SKAT $^{13}$ and WST $^{33}$ (online supplementary table S2). Subsequently, Fisher's exact test, BIN-test and ClusterBurden were applied to our HCM-GnomAD case-control data across the 34 cardiomyopathy gene panels. Post hoc analytic power calculations were performed by treating Clusterburden and Fisher's exact test as likelihood ratio tests, with non-centrality parameters scaling with sample size. ${ }^{3435}$

\section{Hotspot estimation and in silico predictor modelling using GAMs}

To test the hypothesis that a variant's position can improve pathogenicity interpretation, we considered gene-specific models of variant clustering in cases and controls. By combining 
information on gene-level burden and variant positions, these data-driven models estimate the regional burden across the linear protein sequence to quantify mutational hotspots. The models were fitted in the GAM framework, ${ }^{36}$ implemented in the $\mathrm{R}$ package ' $\mathrm{mgcv}$ '. ${ }^{37}$ The outcome variable was disease status, so each model was unsupervised with respect to previous classifications of pathogenicity. The training data included all rare missense variants in cases and controls, including known pathogenic variants in the control set. Therefore, this approach implicitly models incomplete penetrance and benign background variation, leading to unbiased estimates of variant ORs.

GAMs, as an extension of the linear modelling framework, are designed to deal with non-linear relationships of unknown complexity, between explanatory variables (eg, residue position) and the response variable (eg, case-control status). When a relationship is potentially non-linear, it is represented by a smooth curve instead of a straight line. These curves are inferred automatically using restricted maximum likelihood, which reduces overfitting by penalising excessive 'wiggliness'.

Using this framework, we defined the structure of the hotspot model, which models carrier status (gene-level burden) and residue position (clustering). To incorporate gene-level burden, non-carriers must also be modelled. However, as variant-level features such as residue position are meaningless for non-carriers, a nested model structure is required, whereby residue position is included only as an interaction with carrier status. Under these circumstances, the smoothed residue position term is multiplied by zero for non-carriers, excluding this undefined data from the model. The structure of the hotspot model is as follows:

$P=\beta_{0}+\beta_{1}$ carrier_status $+\mathrm{s}_{1}$ (residue_position, by $=$ carrier status) $+\varepsilon$

where $P$ is the probability of being a case, $\beta_{0}$ is the model intercept, $\beta_{1}$ is a linear coefficient for carrier_status, $s_{1}$ is a smoothed (ie, non-linear) function for residue position, by is used to generate factor-smooth interactions and $\varepsilon$ is a binomial error term. To account for uneven sequencing coverage between the cases and controls, the contribution of each datum to the log-likelihood was weighted by the reciprocal of the mean $10 \times$ coverage in the surrounding region (online supplementary method S2).

The feasibility of this approach is dependent on the number of observations, thereby limiting its application in our data to the six core sarcomeric genes: MYBPC3, MYH7, MYL2, MYL3, TNNI3 and TNNT2, each carrying at least 20 rare missense variants. A hotspot model was produced for each gene, and raw model predictions for each residue position, in the form of logistic probabilities and SEs, were transformed to ORs and 95\% CI. There is currently no universal guidance on how to quantitatively apply ACMG criteria PM1. However, using the probability that a variant is a case variant as a proxy of pathogenicity, we can use predicted probabilities to attribute levels of evidence. Here we stratify variants based on the probability thresholds $0.9,0.95$ and 0.99 to represent supporting, moderate and strong evidence of pathogenicity. These correspond to ORs of approximately 10,20 and 100 .

As GAMs are additive in structure, it is straightforward to include further predictors in the model. Here we experimented with the inclusion of variant prediction scores extracted from the dbNSFP4.0 database for nonsynonymous SNPs' functional predictions. ${ }^{38}$ These in silico prediction algorithms are covered by the ACMG criteria PP3, however, like criteria PM1, it is challenging to apply this criterion quantitatively. It is unclear which threshold determines a pathogenic variant with a given probability and whether these thresholds are consistent across genes.
Both of these problems can be solved using gene-specific models, as the relationship between in silico predictors and disease status is inferred. Furthermore, uncertainty on the usage of these scores can be quantified.

To avoid overfitting, a strict two-stage feature selection procedure was implemented. In stage 1 , dbNSFP features (online supplementary figure $\mathrm{S} 3 \mathrm{~A}$ ) with a marginal $\mathrm{p}$ value $<0.002$ were selected (0.05/24 Bonferroni corrected). In stage 2, backwards elimination was implemented, whereby features with the lowest $\mathrm{p}$ value are removed one at a time until all features are significant (Bonferroni corrected for the number of features selected in stage 1). The resulting models (online supplementary figure S3B), which are henceforth termed hotspot + models, assimilate evidence of gene burden, variant clustering and pathogenicity prediction scores.

Model performance for these GAMs are best judged by the estimates of uncertainty accompanying predictions. However, to determine the relative ability of these models to predict pathogenicity, they were compared with models based on single in silico predictors and expert variant classifications. Relative performance was assessed using the receiver operator characteristic area under the curve (AUC) across cross-fold validations performed by dividing the data into 10 random training and test sets using an 80\%:20\% ratio. Hotspots were also compared with

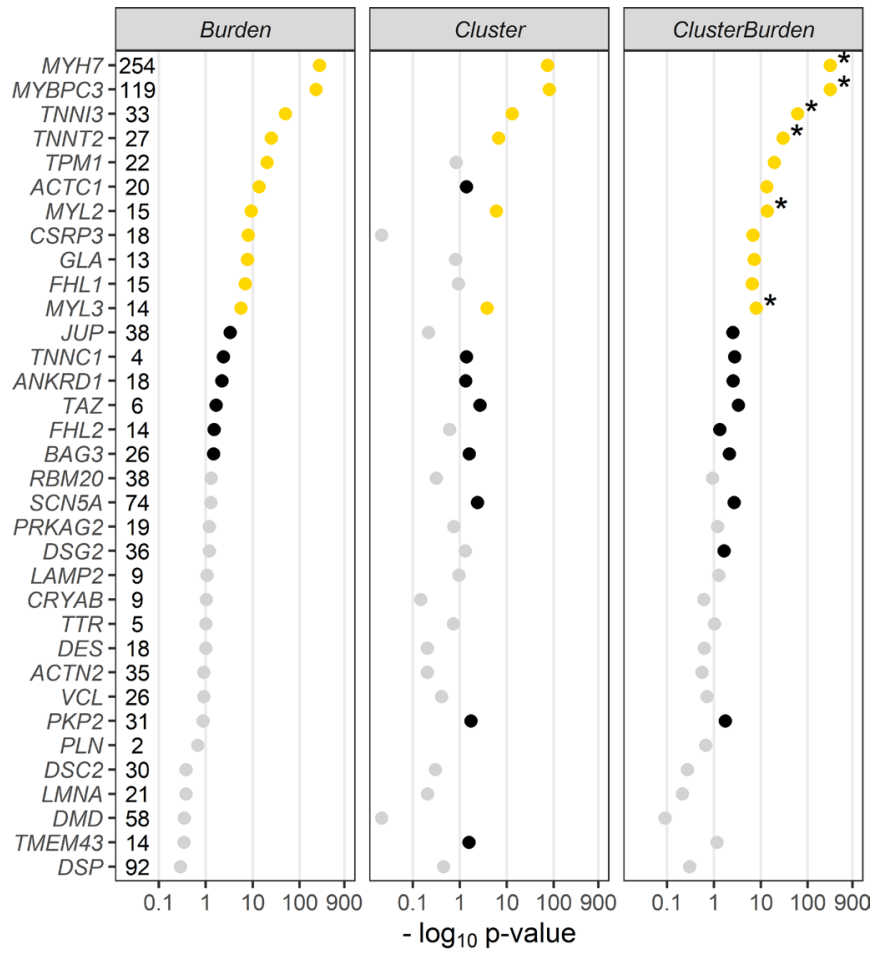

Significance level $\bullet p<0.00049 \bullet p<0.05 \bullet p>0.05$

Figure $1 P$ values $\left(-\log _{10}\right)$ from a Fisher's exact test (Burden), BIN-test (Cluster) and ClusterBurden across a 34-gene cardiomyopathy gene panel. Our case-control dataset contains 5338 hypertrophic cardiomyopathy cases and 125748 GnomAD controls. For all tests, only missense variants with a popmax MAF less than $0.01 \%$ were considered. The number of observed case variants in each gene is displayed next to the gene symbol. $P$ values displayed in yellow are significant after Bonferroni correction for 34 genes $x$ three tests $(p<0.00049)$, $p$ values in black are nominally significant $(p<0.05)$ and $p$ values in grey are insignificant $(p>0.05)$. Asterisks denote genes where the ClusterBurden $\mathrm{p}$ value is lower than the burden $p$ value. 


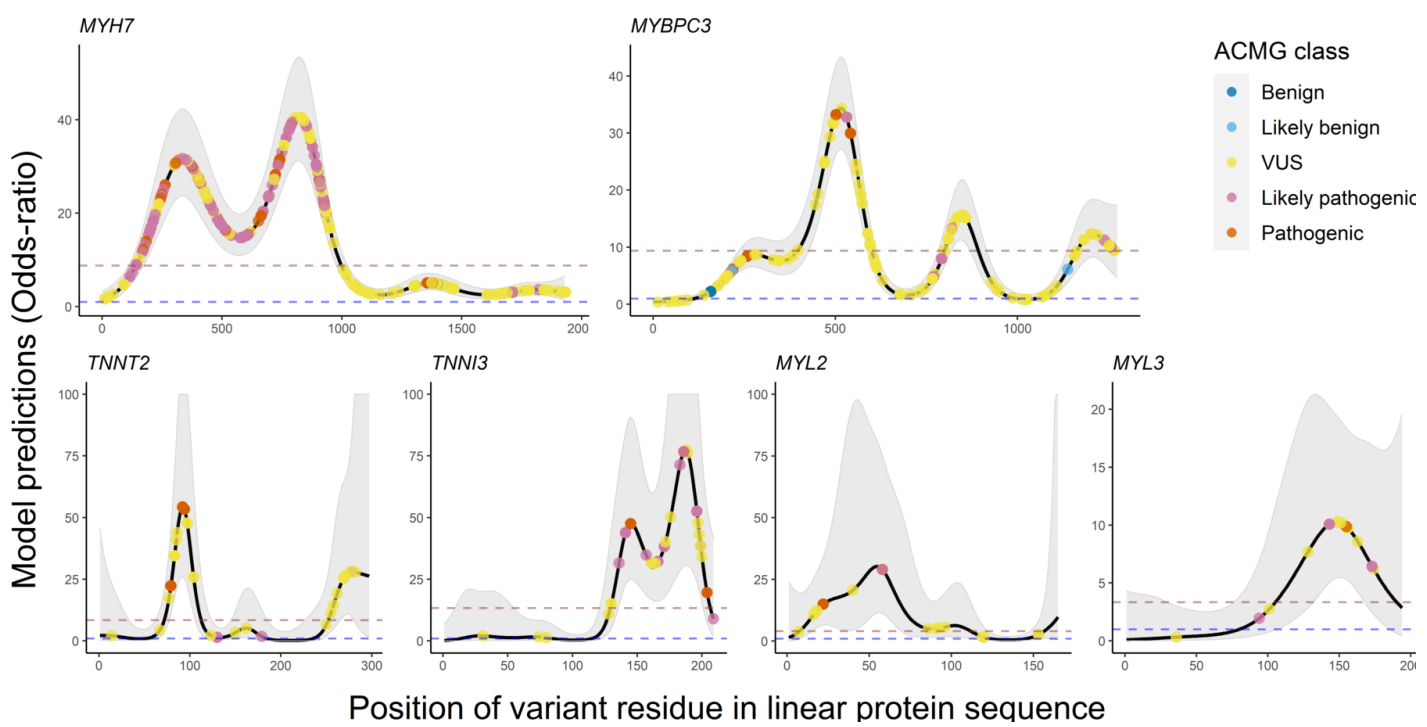

Figure 2 OR predictions and 95\% Cls for hotspot models. Mutational hotspots were modelled for six firmly established HCM disease genes. The $95 \%$ Cls for model predictions are displayed as light grey shading. A dashed blue line at $\mathrm{OR}=1$ indicates the threshold at which a region is in excess in cases or $>1$ or depleted in cases or $<1$. Rare missense variants in the HCM data are superimposed on the predicted model and coloured by their ACMG class assessed by Oxford Medical Genetics Laboratory. ACMG, American College of Medical Genetics and Genomics; HCM, hypertrophic cardiomyopathy; VUS, variant of uncertain significance.

those identified by Walsh et $a l,{ }^{39}$ based on a partially overlapping dataset, using a one-dimensional clustering algorithm (henceforth abbreviated as $1 \mathrm{dC}$ ) and constrained-coding regions (CCR) identified by Havrilla et al. ${ }^{40}$

\section{RESULTS}

\section{Testing the hypothesis of clustered missense variants}

Under the null hypothesis of no excess burden or clustering, the false-positive rate of the BIN-test and $\mathrm{AD}$ test were adequately controlled in simulated data, whereas the KS test was overly conservative (online supplementary table S3). The BIN-test had superior power than $\mathrm{AD}$ or KS under all clustering scenarios and protein lengths with, on average, 1.8-fold more power to detect clustering. As power covaries with the number of observed variants, power was higher for longer proteins as well as proteins with larger pathogenic regions.

Correlations between $\mathrm{p}$ values generated by the BIN-test and Fisher's exact test were compared for simulated data under: (1) a null model of no association or (2) a disease model of overburdened and clustered variants. For the disease model, there was a positive correlation (Spearman's rank correlation rho $=0.40$ ) between $\mathrm{p}$ values, as anticipated as the power of these tests covaries with the number of observed variants. However, under the null model, the $\mathrm{p}$ values were completely uncorrelated $(\mathrm{rho}=0.00, \mathrm{p}=0.5)$, satisfying the independence assumption of Fisher's method.

The false-positive rate for ClusterBurden, DoEstRare, CLUSTER and C-alpha were all well controlled in the simulated datasets (online supplementary table S3). On the contrary, SKAT and WST showed markedly inflated false-positive rates under the null and were not examined further. ClusterBurden was the most powerful method when clustering was present with an average of $72 \%$ power, $3 \%$ higher than the second-best test DoEstRare. The best method under the uniform model (ie, burden-only) was CLUSTER, which had $\sim 5 \%$ more power than ClusterBurden. Among the position-informed RVATs, ClusterBurden was the most rapid to compute taking less than a second per gene, whereas DoEstRare and CLUSTER took over 20 or 4 min, respectively.

We then examined 34 cardiomyopathy genes for rare missense variant associations with Fisher's exact test (burden), BIN-test (cluster) and ClusterBurden (combined cluster and burden) in our cohorts of HCM cases and GnomAD controls (figure 1). Significance thresholds were conservatively Bonferroni adjusted to allow for 34 genes $\times$ three methods (ie, $\mathrm{p}$ values adjusted for 102 tests to $\mathrm{p}<0.00049$ ). Significant burden signals were then detected in 11 genes with Fisher's exact test; MYH7 $(\mathrm{p}<5.84$ $\left.\times 10^{-265}\right)$, MYBPC3 $\left(\mathrm{p}<1.43 \times 10^{-222}\right)$, TNNI3 $(\mathrm{p}<1.96 \times$ $\left.10^{-50}\right)$, TNNT2 $\left(\mathrm{p}<1.08 \times 10^{-25}\right)$, TPM1 $\left(\mathrm{p}<5.79 \times 10^{-21}\right)$, ACTC1 $\left(2.81 \times 10^{-14}\right)$, MYL2 $\left(4.89 \times 10^{-10}\right)$, CSRP3 $(9.29$ $\left.\times 10^{-9}\right)$, GLA $\left(1.77 \times 10^{-8}\right)$, FLH1 $\left(1.32 \times 10^{-7}\right)$ and MYL3 $\left(2.64 \times 10^{-6}\right)$. The BIN-test detected significant clustering for six core sarcomeric genes: MYH7 $\left(\mathrm{p}<1.50 \times 10^{-74}\right)$, MYBPC3 $\left(\mathrm{p}<1.19 \times 10^{-81}\right)$, TNNI3 $\left(\mathrm{p}<9.28 \times 10^{-14}\right)$, TNNT2 $(\mathrm{p}<2.16$ $\left.\times 10^{-7}\right)$, MYL2 $\left(\mathrm{p}<1.08 \times 10^{-6}\right)$, and MYL3 $\left(\mathrm{p}<1.7 \times 10^{-4}\right)$. Two additional sarcomeric genes showed nominal evidence of clustering; ACTC1 $(\mathrm{p}<0.0412)$ and TPM1 $(\mathrm{p}<0.0494)$. ClusterBurden confirmed the association for 11 genes that showed burden signals and calculated substantially lower $\mathrm{p}$ values for the six core-sarcomeric genes with significant clustering. Post hoc power calculations demonstrate the empirical enhanced power for this approach in true disease-causing genes. For example, for MYL2 a 53\% increase and for MYL3 a 52\% increase in sample size would be required for the burden-alone test to have equivalent power to ClusterBurden.

\section{Hotspot and hotspot+ models}

Figure 2 summarises the GAM predictions for six sarcomeric genes in the hotspot models. Visualising the predicted ORs for each residue illuminates the local burden of rare missense variants across each protein, identifying 'mutational-hotspots' and highlighting areas of potential functional importance in HCM pathogenesis. Confidence in these predictions are tight for MYH7 and MYBPC3. Conversely, the genes with fewer observed 


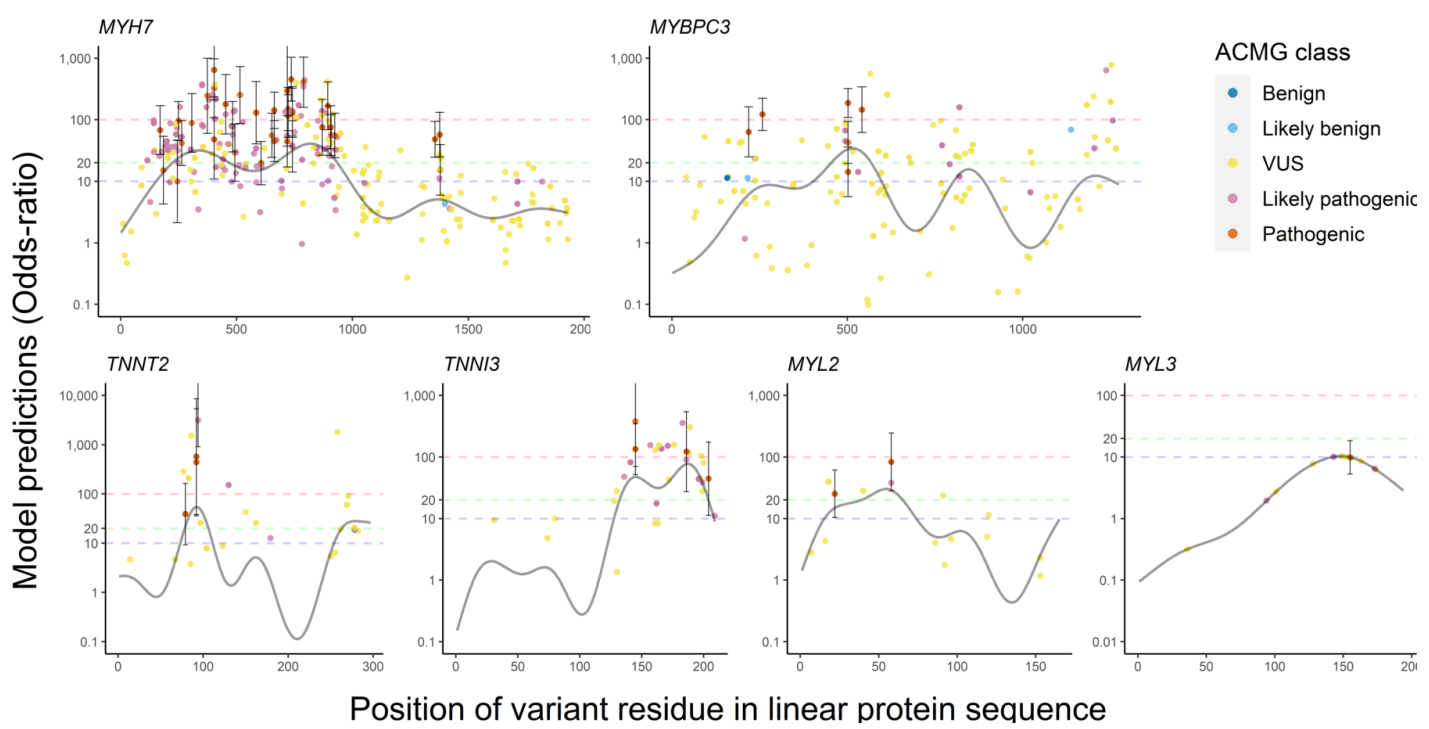

Figure 3 OR predictions and 95\% Cls for hotspot+ models. Points denote rare missense variants in the HCM dataset and are coloured by their ACMG classification assessed by Oxford Medical Genetics Laboratory. ORs on the y-axis are displayed on a $\log _{10}$ scale and were derived from the hotspot+ models; incorporating gene burden, residue position and gene-specific significant secondary features from dbNSFP. The solid black curvy lines represent the predictions for each residue in the protein for a gene burden and position model (hotspot model). Regions above the blue $(O R=10)$, green $(O R=20)$ and red $(O R=100)$ dashed lines ascribe supporting, moderate and strong evidence, respectively, for the combined PM1 and PP3 criteria. ACMG, American College of Medical Genetics and Genomics; HCM, hypertrophic cardiomyopathy; VUS, variant of uncertain significance.

variants have much broader CIs. ORs from all models correlate strongly with expert manually assigned classifications, though there is substantial overlap between classes. Variants with a VUS supplementary figure S1).

Figure 3 displays hotspot+ model predictions (modelling burden, position and in silico predictors) for individual variants in the same six genes. Due to strict feature selection, the number of predictors included in each model depends on the power to detect associations between features and disease status. This resulted in fewer features for genes with fewer observed variants; MYL3 had no additional significant features. As residue position is included as a predictor in each model, predictions generally follow the hotspot model; however, due to additional in silico predictors, ORs tend to vary from this pattern, stratifying risk for variants at the same position.

As with the hotspot models, there was a strong correspondence between predictions and expert classifications (mean rho 0.41 across six models). In MYH7, mean predicted ORs for pathogenic, likely pathogenic and VUS variants observed in cases, were 74, 50 and 20, respectively. Again, the VUS class had the highest heterogeneity, with predicted ORs ranging from 0.25 to 197 (MYH7). Half of these VUSs are observed in a single case and absent in controls (private singletons). The empirical ORs for these variants, based on the case and control frequencies and adding 0.5 to zero-count cells (Haldane continuity correction ${ }^{41}$ ), had wide 95\% CIs: 44.9 (1.5 to 1338.3). However, predicted ORs for such variants could have greater precision with different point estimates depending on the precise amino acid substitution. In MYH7, five of these singleton VUSs had predicted ORs greater than 100 and three had ORs less than 1.

The mean and SD of AUC for 10 crossfold validations summarise overall model performance (figure 4; online supplementary figure S2). The hotspot+ model had a much higher mean AUC than any individual in silico predictor in isolation. With the exception of MYBPC3, the hotspot model classification show the highest spread in predictions (online

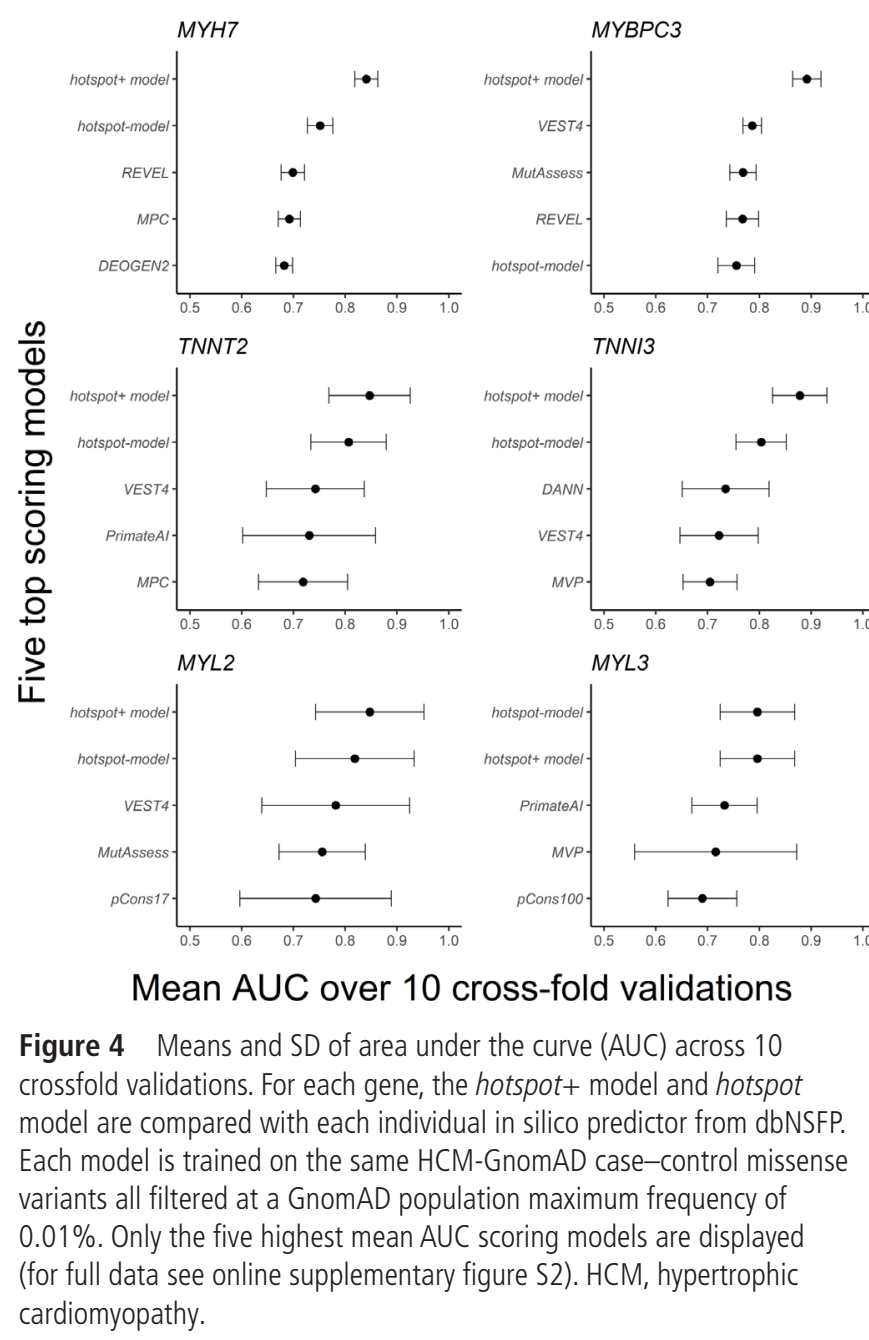


Table 1 Proportion of variants with evidence of pathogenicity in hotspot and hotspot+ models

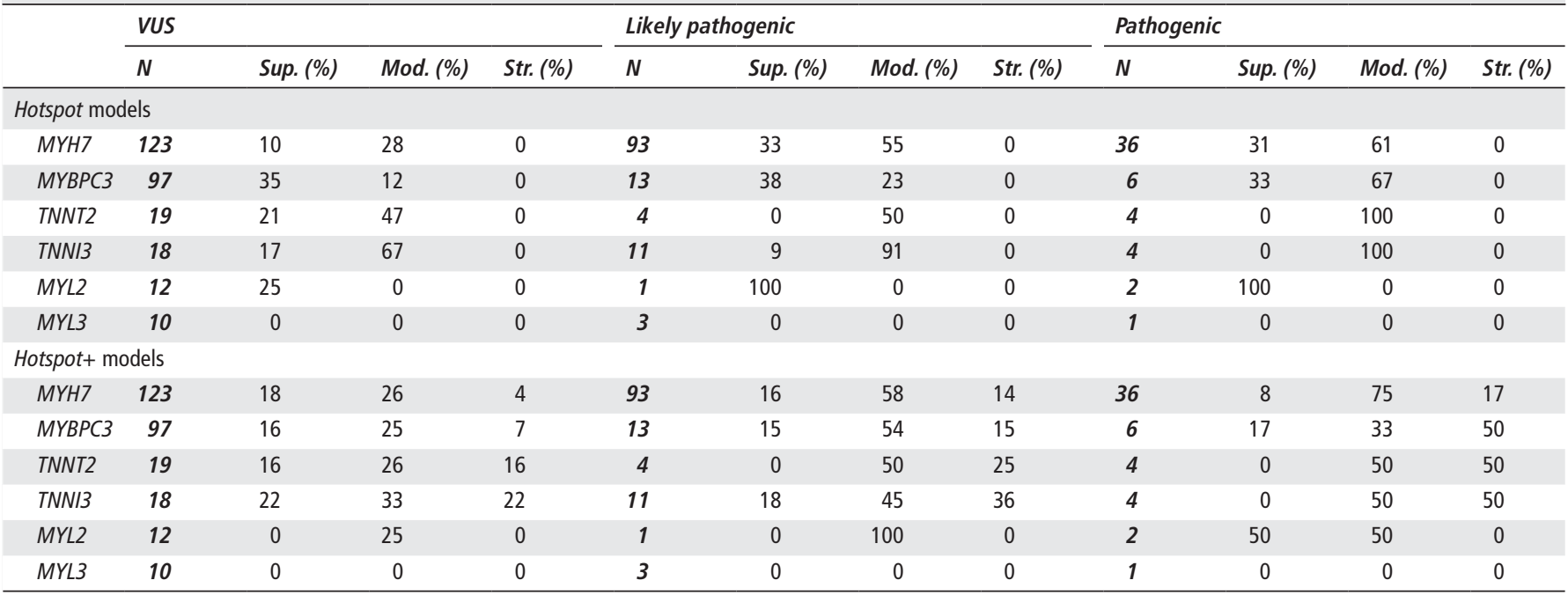

Each observed variant across six genes in our HCM cases was given supporting ( $O R>10$; Sup.), moderate ( $O R>20$; Mod.) or strong (OR $>100$; Str.) evidence of pathogenicity based on model predictions from the hotspot and hotspot+ models. The proportion of variants with supporting, moderate or strong evidence are stratified by expert classifications made by Oxford Medical Genetics Laboratory.

$\mathrm{HCM}$, hypertrophic cardiomyopathy.

outperformed any standalone score from dbNSFP. This suggests that residue position is more important in determining pathogenicity than the in silico predictors in dbNSFP for these sarcomeric proteins. The AUC SD for MYH7 and MYBPC3 were the smallest, suggesting they have the highest capacity to generalise to new variants.

The GAMs were then used to attribute evidence of pathogenicity based on the ACMG criteria PM1 and PP3. Using the ACMG OR thresholds described in the methods, table 1 shows the proportion of variants in our cohort with evidence of pathogenicity predicted by the hotspot and hotspot+ models. For the hotspot model, the PM1 criteria was satisfied, with supporting $(\mathrm{OR}>10)$ or moderate $(\mathrm{OR}>20$ ) evidence, for some variants in all genes except MYL3. Strong evidence of pathogenicity $(\mathrm{OR}>100)$ was not predicted by the hotspot model for any variant. Conversely, the hotspot + model, which combined criteria PM1 and PP3, provided strong evidence of pathogenicity for many variants, including VUSs in $\mathrm{MYH7}$, MYBPC3, TNNT2 and TNNI3.

Linear predictions from the hotspot models were stratified to delineate moderate $(O R \geq 20)$, supporting $(O R \geq 10)$ and weak $(\mathrm{OR} \geq 5)$ regions of pathogenicity potential and compared with $1 \mathrm{dC}$ hotspot regions ${ }^{39}$ and CCRs ${ }^{40}$ (figure 5 ). There was partial cluster overlap for 5/6 genes; however, no cluster was identified by $1 \mathrm{dC}$ for MYL2, and the hotspot model did not identify any clustering in CSRP3. Although some overlap with the clusters and CCRs was present in MYH7 and TNNI3, for the most part, there was weak correspondence between this metric and our observed clusters.

Detailed model prediction statistics for the hotspot and hotspot+ model are presented in online supplementary table S5. A web application, pathogenicity_by_position, is available to facilitate the exploration of the hotspot and hotspot+ models (R Shiny: https://adamwaring.shinyapps. io/Pathogenicity_by_position). Users can explore alternative models and submit their own missense variants to retrieve predicted ORs and support intervals. A further R package is available for cluster detection and association testing using BIN-test and ClusterBurden (https://github.com/adamwaring/ ClusterBurden). A guide to using pathogenicity_by_position is available on the app home page, and instructions for using the $\mathrm{R}$ package are available in the documentation and associated vignettes.

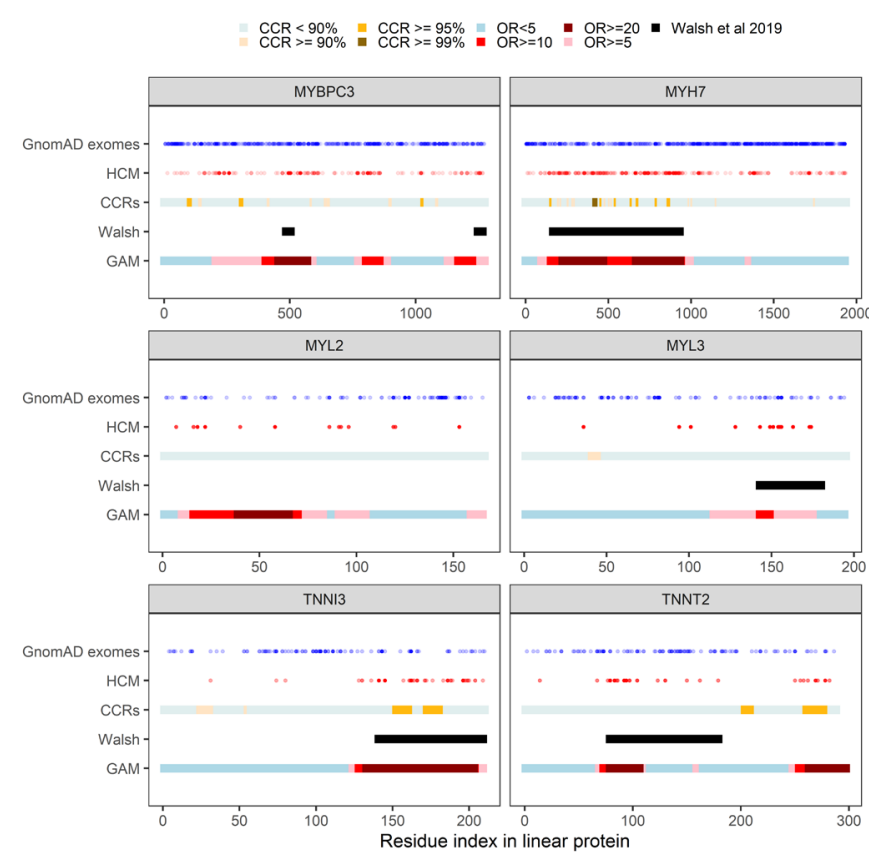

Figure 5 Overlap of GAM hotspots with clusters identified by Walsh et $a l^{39}$, constrained-coding regions identified by Havrilla et $a l^{40}$ and empirical missense variant positions. For six firmly established sarcomeric HCM genes, rare (popmax <0.1\%) missense variants in GnomAD exomes (blue) and HCM (red) are plotted by their position in the linear protein sequence. GAM predictions from the hotspot models are stratified by ORs into moderate $(O R \geq 20)$, supporting $(O R \geq 10)$ and weak $(O R \geq 5)$ evidence clusters. Mutational clusters identified by Walsh et $\mathrm{a}^{39}$ are shown in black. Constrained-coding regions are plotted after stratification by their constraint percentile ( $\geq 99 \%, \geq 95 \%, \geq 90 \%$ and $<90 \%)$. GAM, generalised additive model. 


\section{DISCUSSION}

We present new statistical approaches to incorporate residue position in the analysis of rare missense variants in Mendelian disease genes. Our association tests were well calibrated in simulated data, the BIN-test detected significant clustering in almost all firmly established HCM genes, and ClusterBurden gave superior power over a simple burden test. Datadriven models were applied to six core sarcomeric genes to estimate mutational hotspots and provides a flexible method for quantitative application of ACMG criteria PM1 and PP3. Our results demonstrate that residue position can be a powerful predictor of both gene and variant pathogenicity. Furthermore, GAMs can quantify the statistical uncertainty surrounding the application of in silico algorithms using gene-specific approaches.

BIN-test is a powerful approach to test for variant clustering in known or putative disease genes. ClusterBurden is an RVAT with superior power than traditional methods when pathogenic variants cluster in specific protein regions. Both tests keep false-positives below 5\% and are rapid to compute, making them scalable for whole-exome scanning of very large datasets like the UK Biobank. Although ClusterBurden has slightly reduced power in the absence of clustering, we observed clustering for most well-established HCM genes where missense variants cause disease. Therefore, this method has the potential to be more powerful to detect undiscovered low penetrance genes.

The most significant position signal was observed in the beta myosin heavy chain protein (MYH7: ENST00000355349), driven by a substantial excess in the motor domain, a finding that has been long recognised. ${ }^{42}$ High-case and low-control variant density in the carboxy terminus of this protein might lead an observer to hypothesise a regional protective effect on HCM risk (online supplementary figure S3). In sharp contrast, the GAM model predicts a modestly excessive burden $(\mathrm{OR} \sim 3)$ across this entire region discounting this hypothesis (figure 2).

A strong position signal, driven by four potential clusters in domains C1, C3, C7 and C10, was observed in cardiac myosinbinding protein C (figure 2; MYBPC3: ENST00000545968). The C1 domain is a suspected myosin S2 and actin-binding site and the C10 domain is a possible TTN binding site. ${ }^{43}$ To explore whether the signal was overly driven by highfrequency founder mutations, seven variants with allele counts above 10, p.Arg810His, p.Asp770Asn, p.Glu542Gln, p.Arg502Trp, p.Arg495Gln, p.Glu258Lys and p.Val219Leu (ENST00000545968), were masked in a sensitivity analysis. In their absence, a strong position signal persists $\left(\mathrm{p}<3 \times 10^{-9}\right)$ and remaining peak densities overlap with the locations of the (masked) founder mutations (online supplementary figure S4).

Eighty-nine per cent of 27 case variants in cardiac troponin T (TNNT2: ENST00000509001) map to clusters between residues 67-179 and 250-282 (figure 2). The first cluster overlies a previously reported tropomyosin-binding region ${ }^{44}$ and six variants fall between residues 92-110, a region previously noted to impair tropomyosin-dependent functions. ${ }^{45}$ In cardiac troponin I (TNNI3: ENST00000344887), 91\% of 34 case variants mapped to a cluster spanning residues 128-209. This accords with previous studies documenting carboxyterminus disease variant clustering. ${ }^{46}$ In myosin light chain 2 (MYL2: ENST00000228841), half of 30 case variants cluster between residues 25 and 100, whereas control variants clustered in the C-terminus (figure 2). In myosin light chain 3
(MYL3: ENST00000395869), 79\% of 14 case variants cluster between residues 125 and 175 (figure 2), whereas control variants were uniformly distributed.

GAMs were used to model variant pathogenicity based on mutational hotspots (hotspot model) and a combination of mutational hotspots and in silico predictors (hotspot + model). GAMs have attractive statistical properties, not necessarily shared by other machine-learning approaches, in that they can produce familiar interpretable results via variant-specific ORs and accompanying 95\% CIs. Unlike empirical ORs, based solely on observed frequencies for variants, GAM ORs draw on a much larger pool of information. This permits the estimation of variant-specific ORs whenever the empirical frequencies are uninformative. Furthermore, as the response variable is case status, models are unbiased by previous classifications and account for both penetrance and background rare variation.

Reassuringly, model predictions were positively correlated with expert manually curated classifications. Using a probabilistic approach, we attributed different levels of evidence for the criteria PM1 and PP3. Currently for HCM, criteria PM1 is only applied consistently to MYH7 as moderate evidence for variants that fall in the residue 181-937 motor domain. ${ }^{20}$ Hotspot models extend this criteria to five more sarcomeric genes and stratify evidence as supporting $(\mathrm{OR} \geq 10)$ or moderate $(\mathrm{OR} \geq 20)$. When in silico predictors were included in the model, evidence was occasionally strong ( $O R \geq 100)$. This relies on collapsing two ACMG criteria into one, a relevant modification of the current additive guidelines. ${ }^{16}$

Constrained coding regions percentiles ${ }^{40}$ have been calculated across the exome using GnomAD variant data. Intraspecies constraint does not appear to be a definitive metric for the identification of mutational hotspots in HCM. CCRs may be primarily correlated with regions linked to extreme consequences such as embryonic lethality or at least diseases more severe than HCM. Alternatively, the mismatch could be driven by incomplete penetrance, obscuring the constraint in these regions.

Walsh et $\mathrm{al}^{39}$ employed a $1 \mathrm{dC}$ to detect local intracohort enrichment of variants with a binomial test. Inconsistent hotspot assignments between 1dC and GAM are likely attributable to small-sample variation, notably for genes with few variants such as MYL2. However, there are conceptual differences. The most impactful of these is that $1 \mathrm{dC}$ locates clusters using a case-only scan, whereas the hotspot models compare cases and controls. Potential consequences of the case-only approach include reduced power to detect multiple clusters as 'outside-window' counts are enriched. Furthermore, the GAM approach provides more refined per-residue estimates of pathogenicity, allowing stratification into multiple pathogenicity bands.

In contrast to previous data-driven HCM analyses based on aggregations of clinical reports, adjustment for uneven sequence coverage for the HCM samples was possible due to the availability of BAM files. However, incomplete coverage control is a limitation of this study and more sophisticated adjustment methods may refine hotspot estimation. As a data-driven modelling approach, the GAM estimates become increasingly refined as more data becomes available. Additional improvements to the modelling framework could include the incorporation of historical clustering data as Bayesian priors to further reduce uncertainty in model estimates. Although applied here to HCM as an exemplar proof of concept, ongoing work seeks to extend this method more 
broadly to Mendelian disease genes with adequate cohort sizes and suitable levels of genetic heterogeneity.

\section{CONCLUSIONS}

We present a rare disease/rare variant association test that shows higher theoretical power in synthetic data than traditional burden testing for Mendelian diseases and empirically enhanced power for six sarcomeric HCM genes. We demonstrate how a flexible statistical modelling approach can simultaneously quantify burden and mutational hotspots, with application to firmly established HCM genes. Our approach extends previous studies that defined discrete regions of increased pathogenicity potential to develop a refined map of regional burden across proteins. With the addition of annotation information as covariates in the model, when in silico predictors are used alongside burden and positional information, unique variant-level predictions can outperform published meta-predictors with enhanced sensitivity and specificity.

\section{Twitter Adam Waring @Adam_Waring_}

Acknowledgements We would like to acknowledge Anuj Goel for his bioinformatics support in data curation and Michael Bowman for his support in accessing data from the clinical genetics laboratory. Computation used the Oxford Biomedical Research Computing (BMRC) facility, a joint development between the Wellcome Centre for Human Genetics and the Big Data Institute supported by Health Data Research UK and the NIHR Oxford Biomedical Research Centre.

Contributors AW and MF led conception and design of work. AW contributed to data curation, conducted all analyses, developed statistical methods and associated software and wrote each draft of manuscript. MF supervised all analyses and interpretation of results. AH led the curation and quality control of the hypertrophic cardiomyopathy datasets. SS contributed to quality control of the data. CK and SN supported access to the HCMR dataset. KT and HW advised and guided the clinical aspects of the work. MF and KT reviewed and editing each draft of the manuscript

Funding Wellcome Trust doctoral studentship (203834/Z/16/Z) to AW, MRC doctoral studentship to AH, Welcome Trust core award (203141/Z/16/Z, MF and $H W)$, the Oxford BHF Centre of Research Excellence (RE/13/1/30181, MF and HW), HW has received support from the National Institute for Health Research Oxford Biomedical Research Centre. CK, SN and HW received support from a National Heart, Lung, and Blood Institute (grant U01HL117006-01A1)

Disclaimer The views expressed are those of the author(s) and not necessarily those of the NHS, the NIHR or the Department of Health.

Competing interests None declared.

Patient consent for publication Not required.

Ethics approval The research protocol was approved by the South Central Oxford A Research Ethics Committee (REC reference: 14/SC/0190); written informed consent was obtained from all participants.

Provenance and peer review Not commissioned; externally peer reviewed.

Data availability statement Data are available on reasonable request. Due to the confidential nature of some of the research materials supporting this publication, not all of the data can be made accessible to other researchers. Please contact the corresponding author for more information.

Open access This is an open access article distributed in accordance with the Creative Commons Attribution 4.0 Unported (CC BY 4.0) license, which permits others to copy, redistribute, remix, transform and build upon this work for any purpose, provided the original work is properly cited, a link to the licence is given, and indication of whether changes were made. See: https://creativecommons.org/ licenses/by/4.0/

ORCID iDs

Adam Waring http://orcid.org/0000-0002-3590-2560

Andrew Harper http://orcid.org/0000-0001-5327-0328

\section{REFERENCES}

1 Bissler JJ, Cicardi M, Donaldson VH, Gatenby PA, Rosen FS, Sheffer AL, Davis AE. A cluster of mutations within a short triplet repeat in the $\mathrm{C} 1$ inhibitor gene. Proc Nat Acad Sci U S A 1994;91:9622-5.
2 Robertson SP, Twigg SRF, Sutherland-Smith AJ, Biancalana V, Gorlin RJ, Horn D, Kenwrick SJ, Kim CA, Morava E, Newbury-Ecob R, Orstavik KH, Quarrell OWJ, Schwartz CE, Shears DJ, Suri M, Kendrick-Jones J, Wilkie AOM, OPD-spectrum Disorders Clinical Collaborative Group. Localized mutations in the gene encoding the cytoskeletal protein filamin a cause diverse malformations in humans. Nat Genet 2003:33:487-91.

3 Hunt KA, Zhernakova A, Turner G, Heap GAR, Franke L, Bruinenberg M, Romanos J, Dinesen LC, Ryan AW, Panesar D, Gwilliam R, Takeuchi F, McLaren WM, Holmes GKT, Howdle PD, Walters JRF, Sanders DS, Playford RJ, Trynka G, Mulder CJJ, Mearin ML, Verbeek WHM, Trimble V, Stevens FM, O'Morain C, Kennedy NP, Kelleher D, Pennington DJ, Strachan DP, McArdle WL, Mein CA, Wapenaar MC, Deloukas P, McGinnis R, McManus R, Wijmenga C, van Heel DA. Newly identified genetic risk variants for celiac disease related to the immune response. Nat Genet 2008;40:395-402.

4 Henderson DM, Lee A, Ervasti JM. Disease-causing missense mutations in actin binding domain 1 of dystrophin induce thermodynamic instability and protein aggregation. Proc Nat/ Acad Sci U SA 2010;107:9632-7.

5 Fine DM, Wasser WG, Estrella MM, Atta MG, Kuperman M, Shemer R, Rajasekaran A, Tzur S, Racusen LC, Skorecki K. APOL1 risk variants predict histopathology and progression to ESRD in HIV-related kidney disease. J Am Soc Nephrol 2012;23:343-50

6 Lelieveld SH, Wiel L, Venselaar H, Pfundt R, Vriend G, Veltman JA, Brunner HG, Vissers LELM, Gilissen C. Spatial clustering of de novo missense mutations identifies candidate neurodevelopmental disorder-associated genes. Am J Hum Genet 2017;101:478-84.

7 Persyn E, Karakachoff M, Le Scouarnec S, Le Clézio C, Campion D, Consortium FE, Schott J-J, Redon R, Bellanger L, Dina C. DoEstRare: a statistical test to identify local enrichments in rare genomic variants associated with disease. PLoS One 2017;12:e0179364

8 Collins FS. Positional cloning moves from perditional to traditional. Nat Genet 1995:9:347-50.

9 Walsh R, Thomson KL, Ware JS, Funke BH, Woodley J, McGuire KJ, Mazzarotto F, Blair E, Seller A, Taylor JC, Minikel EV, MacArthur DG, Farrall M, Cook SA, Watkins H. Reassessment of Mendelian gene pathogenicity using 7,855 cardiomyopathy cases and 60,706 reference samples. Genet Med 2017:19:192-203.

10 Guo MH, Plummer L, Chan Y-M, Hirschhorn JN, Lippincott MF. Burden testing of rare variants identified through exome sequencing via publicly available control data. Am J Hum Genet 2018;103:522-34.

11 Curtis D. A rapid method for combined analysis of common and rare variants at the level of a region, gene, or pathway. Adv Appl Bioinform Chem 2012;5:1-9.

12 Curtis D. A weighted burden test using logistic regression for integrated analysis of sequence variants, copy number variants and polygenic risk score. Eur J Hum Genet 2019;27:114-24

13 Wu MC, Lee S, Cai T, Li Y, Boehnke M, Lin X. Rare-variant association testing for sequencing data with the sequence kernel association test. Am J Hum Genet 2011:89:82-93.

14 Neale BM, Rivas MA, Voight BF, Altshuler D, Devlin B, Orho-Melander M, Kathiresan $\mathrm{S}$, Purcell SM, Roeder K, Daly MJ, Daly K. Testing for an unusual distribution of rare variants. PLoS Genet 2011;7:e1001322.

15 Lin W-Y. Association testing of clustered rare causal variants in case-control studies. PLoS One 2014;9:e94337.

16 Richards S, Aziz N, Bale S, Bick D, Das S, Gastier-Foster J, Grody WW, Hegde M, Lyon E, Spector E, Voelkerding K, Rehm HL, ACMG Laboratory Quality Assurance Committee. Standards and guidelines for the interpretation of sequence variants: a joint consensus recommendation of the American College of medical genetics and genomics and the association for molecular pathology. Genet Med 2015;17:405-23.

17 Ghosh R, Oak N, Plon SE. Evaluation of in silico algorithms for use with ACMG/AMP clinical variant interpretation guidelines. Genome Biol 2017;18:225.

18 Watkins H, Ashrafian H, Redwood C. Inherited cardiomyopathies. N Engl I Med 2011;364:1643-56.

19 Rehm HL, Berg JS, Brooks LD, Bustamante CD, Evans JP, Landrum MJ, Ledbetter DH, Maglott DR, Martin CL, Nussbaum RL, Plon SE, Ramos EM, Sherry ST, Watson MS, ClinGen. ClinGen--the Clinical Genome Resource.. N Engl J Med 2015;372:2235-42.

20 Kelly MA, Caleshu C, Morales A, Buchan J, Wolf Z, Harrison SM, Cook S, Dillon MW, Garcia J, Haverfield E, Jongbloed JDH, Macaya D, Manrai A, Orland K, Richard G, Spoonamore K, Thomas M, Thomson K, Vincent LM, Walsh R, Watkins H, Whiffin N, Ingles J, van Tintelen JP, Semsarian C, Ware JS, Hershberger R, Funke B. Adaptation and validation of the ACMG/AMP variant classification framework for MYH7associated inherited cardiomyopathies: recommendations by ClinGen's inherited cardiomyopathy expert panel. Genet Med 2018:20:351-9.

21 Gelb B, Cavé H, Dillon MW, Gripp KW, Lee J, Mason-Suares H, Rauen K, Williams B, Zenker M, Vincent L. ClinGen RASopathy Working Group. ClinGen's RASopathy expert panel consensus methods for variant interpretation. Genet Med 2018;20:1334-45.

22 Mester JL, Ghosh R, Pesaran T, Huether R, Karam R, Hruska KS, Costa HA, Lachlan K, Ngeow J, Barnholtz-Sloan J, Sesock K, Hernandez F, Zhang L, Milko L, Plon SE, Hegde $M$, Eng C. Gene-Specific criteria for PTEN variant curation: recommendations from the ClinGen PTEN expert panel. Hum Mutat 2018:39:1581-92. 
23 Oza A, DiStefano M, Hemphill S, Cushman B, Grant A, Siegert R, Shen J, Chapin A, Boczek N, Schimmenti L, Murry J, Hasadsri L, Nara K, Kenna M, Booth K, Azaiez H, Griffith A, Avraham K, Kremer H, Rehm H, Amr S, Abou Tayoun A. ClinGen hearing loss clinical domain Working Group. expert specification of the ACMG/AMP variant interpretation guidelines for genetic hearing loss. Hum Mutat 2018;39:1593-613.

24 Lek M, Karczewski KJ, Minikel EV, Samocha KE, Banks E, Fennell T, O’Donnell-Luria AH, Ware JS, Hill AJ, Cummings BB, Tukiainen T, Birnbaum DP, Kosmicki JA, Duncan LE, Estrada K, Zhao F, Zou J, Pierce-Hoffman E, Berghout J, Cooper DN, Deflaux N, DePristo M, Do R, Flannick J, Fromer M, Gauthier L, Goldstein J, Gupta N, Howrigan D, Kiezun A, Kurki MI, Moonshine AL, Natarajan P, Orozco L, Peloso GM, Poplin R, Rivas MA, Ruano-Rubio V, Rose SA, Ruderfer DM, Shakir K, Stenson PD, Stevens C, Thomas BP, Tiao G, Tusie-Luna MT, Weisburd B, Won H-H, Yu D, Altshuler DM, Ardissino D, Boehnke M, Danesh J, Donnelly S, Elosua R, Florez JC, Gabriel SB, Getz G, Glatt SJ, Hultman CM, Kathiresan S, Laakso M, McCarroll S, McCarthy MI, McGovern D, McPherson R, Neale BM, Palotie A, Purcell SM, Saleheen D, Scharf JM, Sklar P, Sullivan PF, Tuomilehto J, Tsuang MT, Watkins HC, Wilson JG, Daly MJ, MacArthur DG, Exome Aggregation Consortium. Analysis of protein-coding genetic variation in 60,706 humans. Nature 2016;536:285-91.

25 Kramer CM, Appelbaum E, Desai MY, Desvigne-Nickens P, DiMarco JP, Friedrich MG, Geller N, Heckler S, Ho CY, Jerosch-Herold M, Ivey EA, Keleti J, Kim D-Y, Kolm P, Kwong RY, Maron MS, Schulz-Menger J, Piechnik S, Watkins H, Weintraub WS, Wu P, Neubauer S. Hypertrophic cardiomyopathy registry: the rationale and design of an international, observational study of hypertrophic cardiomyopathy. Am Heart J 2015;170:223-30.

26 Manichaikul A, Mychaleckyj JC, Rich SS, Daly K, Sale M, Chen W-M. Robust relationship inference in genome-wide association studies. Bioinformatics 2010;26:2867-73.

27 Fisher RA. Statistical methods for research workers. Edinburgh: Oliver and Boyd, 1925.

28 Spearman C. The proof and measurement of association between two things. Am J Psychol 1904;15:72-101.

29 Mann H, Wald A. On the choice of the number and width of classes for the chi-square test of goodness of fit. Ann Math Stat 1942;13:306-17.

30 Anderson TW, Darling DA. Asymptotic Theory of Certain "Goodness of Fit" Criteria Based on Stochastic Processes. Ann Math Statist 1952;23:193-212.

31 Kolmogorov AN. Sulla Determinazione Empirica di Una Legge di Distribuzione. Giornale dell'Istituto Italiano degli Attuari 1933;4:83-91.
32 Davison A, Hinkley D. Bootstrap methods and their application (Cambridge series in statistical and probabilistic mathematics. Cambridge: Cambridge University Press, 1997.

33 Madsen BE, Browning SR. A groupwise association test for rare mutations using a weighted sum statistic. PLoS Genet 2009;5:e1000384.

34 Liu BH. Statistical genomics: linkage, mapping, and QTL analysis. CRC press, 1997. 35 Agresti A. Categorical data analysis. New York: John Wiley \& Sons, 1996.

36 Hastie T, Tibshirani R. Generalized additive models. London: Chapman \& Hall, 1990.

37 Wood SN. Fast stable restricted maximum likelihood and marginal likelihood estimation of semiparametric generalized linear models. J Roy Stat Soc 2011;73:3-36.

38 Liu X, Jian X, Boerwinkle E. dbNSFP: a lightweight database of human nonsynonymous SNPs and their functional predictions. Hum Mutat 2011;32:894-9.

39 Walsh R, Mazzarotto F, Whiffin N, Buchan R, Midwinter W, Wilk A, Li N, Felkin L, Ingold N, Govind R, Ahmad M, Mazaika E, Allouba M, Zhang X, de Marvao A, Day SM, Ashley E, Colan SD, Michels M, Pereira AC, Jacoby D, Ho CY, Thomson KL, Watkins H, Barton PJR, Olivotto I, Cook SA, Ware JS. Quantitative approaches to variant classification increase the yield and precision of genetic testing in Mendelian diseases: the case of hypertrophic cardiomyopathy. Genome Med 2019;11:5.

40 Havrilla JM, Pedersen BS, Layer RM, Quinlan AR. A map of constrained coding regions in the human genome. Nat Genet 2019;51:88-95.

41 Haldane JB. The estimation and significance of the logarithm of a ratio of frequencies. Ann Hum Genet 1956;20:309-11.

42 Watkins H, Rosenzweig A, Hwang DS, Levi T, McKenna W, Seidman CE, Seidman JG. Characteristics and prognostic implications of myosin missense mutations in familial hypertrophic cardiomyopathy. N Engl J Med 1992;326:1108-14.

43 Flashman E, Redwood C, Moolman-Smook J, Watkins H. Cardiac myosin binding protein C: its role in physiology and disease. Circ Res 2004;94:1279-89.

44 Pearlstone JR, Smillie LB. The binding site of skeletal alpha-tropomyosin on troponin-T. Can J Biochem 1977;55:1032-8.

45 Palm T, Graboski S, Hitchcock-DeGregori SE, Greenfield NJ. Disease-causing mutations in cardiac troponin T: identification of a critical tropomyosin-binding region. Biophys $J$ 2001;81:2827-37.

46 Mogensen J, Murphy RT, Kubo T, Bahl A, Moon JC, Klausen IC, Elliott PM, McKenna WJ. Frequency and clinical expression of cardiac troponin I mutations in 748 consecutive families with hypertrophic cardiomyopathy. J Am Coll Cardiol 2004;44:2315-25. 